\title{
Periodic solutions for a class of second order delay differential systems
}

\section{Qiong Meng* and Juntang Ding}

\section{"Correspondence:} mengqiong@qq.com School of Mathematical Science, Shanxi University, Taiyuan, Shanxi 030006, P.R. China

\section{Abstract \\ Consider the periodic boundary value problem

$$
\left\{\begin{array}{l}
\ddot{u}(t)+r u(t-\tau)+\nabla F(t, u(t-\tau))=0, \quad \text { a.e. } t \in[0,2 \tau] \\
u(0)-u(2 \tau)=\dot{u}(0)-\dot{u}(2 \tau)=0,
\end{array}\right.
$$

where $\tau>0$ is a given constant, $r \in \mathbf{R}$ is a parameter. Periodic solutions are obtained by using linking theorems.

MSC: 34K13; 34K18; 58E50

Keywords: periodic boundary value problem; delay differential systems; linking theorems

\section{Introduction}

Consider the periodic boundary value problem

$$
\left\{\begin{array}{l}
\ddot{u}(t)+r u(t-\tau)+\nabla F(t, u(t-\tau))=0, \quad \text { a.e. } t \in[0,2 \tau] \\
u(0)-u(2 \tau)=\dot{u}(0)-\dot{u}(2 \tau)=0
\end{array}\right.
$$

where $\tau>0$ is a given constant, $r \in \mathbf{R}$ is a parameter. $F:[0,2 \tau] \times \mathbf{R}^{N} \rightarrow R$ satisfies the following assumptions:

(A) $F(t, x)$ is measurable with respect to $t$, for all $x \in \mathbf{R}^{N}$, continuously differentiable in $x$, for a.e. $t \in[0,2 \tau]$, and there exist $a \in C\left(\mathbf{R}^{+} ; \mathbf{R}^{+}\right)$and $b \in L^{1}\left([0,2 \tau] ; \mathbf{R}^{+}\right)$such that

$$
|F(t, x)|,|\nabla F(t, x)| \leq a(|x|) b(t)
$$

for all $x \in \mathbf{R}^{N}$ and a.e. $t \in[0,2 \tau]$.

Variational methods are very powerful techniques in nonlinear analysis and are extensively used in many disciplines of pure and applied mathematics, including ordinary and partial differential equations, mathematical physics, and geometrical analysis. The existence and multiplicity of solutions for Hamilton systems and Schrödinger equations have been studied extensively via critical point theory; see [1-8].

In the past several years, some results on the existence of periodic solutions for the functional differential equation by the critical point theory have been obtained (see [9-18]). In

(c) 2016 Meng and Ding. This article is distributed under the terms of the Creative Commons Attribution 4.0 International License (http://creativecommons.org/licenses/by/4.0/), which permits unrestricted use, distribution, and reproduction in any medium, provided you give appropriate credit to the original author(s) and the source, provide a link to the Creative Commons license, and indicate if changes were made. 
[16], the authors obtained the multiplicity results for periodic solutions to (1.1) by using critical point theory. Our proof method in this paper is different from the literature [16].

Motivated by the above observation, in this paper, we study the existence of periodic solutions to the system (1.1). The following theorems are the main results of our paper.

(H1) There exists a constant $R_{1}>0$ such that

(i) $F(t, x) \geq 0, \forall|x| \leq R_{1}, t \in[0,2 \tau]$; or

(ii) $F(t, x) \leq 0, \forall|x| \leq R_{1}, t \in[0,2 \tau]$.

(H2) $\lim _{|x| \rightarrow \infty} \inf \frac{F(t, x)}{|x|^{2}}>0$ uniformly for $t \in[0,2 \tau]$.

(H3) $\lim _{|x| \rightarrow 0} \frac{|\nabla F(t, x)|}{|x|}=0$ uniformly for $t \in[0,2 \tau]$.

(H4) There exist $\alpha>1$ and $a>0$ such that

$$
|\nabla F(t, x)| \leq a\left(|x|^{\alpha}+1\right), \quad \forall(t, x) \in[0,2 \tau] \times \mathbf{R}^{N} .
$$

(H5) There exist $\mu>2,0<\beta<2, R>0$, and a function $b(t) \in L^{1}\left([0,2 \tau] ; \mathbf{R}^{+}\right)$such that

$$
\mu F(t, x) \leq(\nabla F(t, x), x)+b(t)|x|^{\beta}, \quad \forall|x| \geq R, t \in[0,2 \tau] .
$$

(H5') There exist $\beta>\alpha \geq 1$ and $b>0, R>0$ such that

$$
(\nabla F(t, x), x)-2 F(t, x) \geq b|x|^{\beta}, \quad \forall|x| \geq R, t \in[0,2 \tau] .
$$

Theorem 1.1 Assume that (H1)-(H5) hold, if 0 is an eigenvalue of $L$ ( $L$ is defined in Section 2), $B V P(1.1)$ has at least one nontrivial solution.

Theorem 1.2 Assume that $(\mathrm{H} 1)-(\mathrm{H} 4)$ and $\left(\mathrm{H} 5^{\prime}\right)$ hold, if 0 is an eigenvalue of $L$ ( $L$ is defined in Section 2), BVP (1.1) has at least one nontrivial solution.

Remark 1.1 The condition (H5) and the condition (H5') are appeared, respectively, in [7] and [8].

\section{Preliminaries}

In order to seek $2 \tau$-periodic orbits of (1.1), let us transform (1.1) to

$$
\left\{\begin{array}{l}
\ddot{u}(t)+r \lambda^{2} u(t-\pi)+\lambda^{2} \nabla F(\lambda t, u(t-\pi))=0, \quad \text { a.e. } t \in[0,2 \pi], \\
u(0)-u(2 \pi)=\dot{u}(0)-\dot{u}(2 \pi)=0,
\end{array}\right.
$$

by making the change of variable $t \rightarrow \frac{\pi}{\tau} t=\lambda^{-1} t$, which implies that a $2 \pi$-periodic solution of (2.1) corresponds to a $2 \tau$-periodic solution of (1.1). Hence we will only look for the $2 \pi$ periodic solutions of (2.1) in the sequel.

Let $L^{2}\left(S^{1}, \mathbf{R}^{N}\right)$ be the space of square integrable $2 \pi$-periodic vector-valued functions with dimension $\mathbf{N}$, and $C^{\infty}\left(S^{1}, \mathbf{R}^{N}\right)$ be the space of $2 \pi$-periodic $C^{\infty}$ vector-valued functions with dimension $\mathbf{N}$. For any $u \in C^{\infty}\left(S^{1}, \mathbf{R}^{N}\right)$, it has the following Fourier expansion in the sense that it is convergent in the space $L^{2}\left(S^{1}, \mathbf{R}^{N}\right)$ :

$$
u(t)=\frac{a_{0}}{\sqrt{2 \pi}}+\frac{1}{\sqrt{\pi}} \sum_{j=1}^{+\infty}\left(a_{j} \cos j t+b_{j} \sin j t\right)
$$


where $a_{0}, a_{j}, b_{j} \in \mathbf{R}^{N}$. Let $H^{1}\left(S^{1}, \mathbf{R}^{N}\right)$ be the closure of $C^{\infty}\left(S^{1}, \mathbf{R}^{N}\right)$ with respect to the Hilbert norm

$$
\|u\|=\left[\left|a_{0}\right|^{2}+\sum_{j=1}^{+\infty}\left(1+j^{2}\right)\left(\left|a_{j}\right|^{2}+\left|b_{j}\right|^{2}\right)\right]^{1 / 2} .
$$

More specifically, $H^{1}\left(S^{1}, \mathbf{R}^{N}\right)=\left\{u \in L^{2}\left(S^{1}, \mathbf{R}^{N}\right):\|u\|<+\infty\right\}$ with the inner product

$$
\langle u, v\rangle=\int_{0}^{2 \pi}[(u(t), v(t))+(\dot{u}(t), \dot{v}(t))] d t
$$

for any $u, v \in H^{1}\left(S^{1}, \mathbf{R}^{N}\right)$, where (., $\left.\cdot\right)$ denotes the usual inner product in $\mathbf{R}^{N}$. The norm on $H^{1}\left(S^{1}, \mathbf{R}^{N}\right)$ is defined by

$$
\|u\|=\left[\int_{0}^{2 \pi}\left(|u(t)|^{2}+|\dot{u}(t)|^{2}\right) d t\right]^{1 / 2} .
$$

It is well known that $H^{1}\left(S^{1}, \mathbf{R}^{N}\right)$ is compactly embedded in $C\left(S^{1}, \mathbf{R}^{N}\right)$ (see Proposition 1.2 of [15]). Define two operators $L_{0}$ and $L_{1}$ from $H^{1}\left(S^{1}, \mathbf{R}^{N}\right)$ into $H^{*}\left(S^{1}, \mathbf{R}^{N}\right)$ as follows: for any $u \in H^{1}\left(S^{1}, \mathbf{R}^{N}\right)$, which are given by

$$
\begin{aligned}
& \left(L_{0} u\right)(v)=\int_{0}^{2 \pi}(\dot{u}(t+\pi), \dot{v}(t)) d t, \\
& \left(L_{1} u\right)(v)=-r \lambda^{2} \int_{0}^{2 \pi}(u(t), v(t)) d t,
\end{aligned}
$$

for all $v \in H^{*}\left(S^{1}, \mathbf{R}^{N}\right)$, where $\dot{u}(t)$ and $H^{*}\left(S^{1}, \mathbf{R}^{N}\right)$ denote the weak derivative of $u$ and the dual space of $H^{1}\left(S^{1}, \mathbf{R}^{N}\right)$, respectively. By the Riesz representation theorem, we can identify $H^{*}\left(S^{1}, \mathbf{R}^{N}\right)$ with $H^{1}\left(S^{1}, \mathbf{R}^{N}\right)$. Thus, $L_{i} u$ can also be viewed as an element belonging to $H^{1}\left(S^{1}, \mathbf{R}^{N}\right)$ such that $\left\langle L_{i} u, v\right\rangle=\left(L_{i} u\right)(v)$ for all $u, v \in H^{1}\left(S^{1}, \mathbf{R}^{N}\right)$, where $i=0$ or 1 . It is easy to check that $L_{0}$ and $L_{1}$ are bounded linear operators on $H^{1}\left(S^{1}, \mathbf{R}^{N}\right)$. Note that $H^{1}\left(S^{1}, \mathbf{R}^{N}\right)$ is compactly embedded in $C\left(S^{1}, \mathbf{R}^{N}\right)$, thus $L_{1}$ is compact on $H^{1}\left(S^{1}, \mathbf{R}^{N}\right)$.

Lemma 2.1 [16] The operator $L:=L_{0}+L_{1}$, that is,

$$
\langle L u, v\rangle=(L u)(v)=\int_{0}^{2 \pi}(\dot{u}(t+\pi), \dot{v}(t)) d t-r \lambda^{2} \int_{0}^{2 \pi}(u(t), v(t)) d t
$$

is self-adjoint on $H^{1}\left(S^{1}, \mathbf{R}^{N}\right)$. The operators $L_{0}$ and $L_{1}$ are also self-adjoint on $H^{1}\left(S^{1}, \mathbf{R}^{N}\right)$.

Lemma 2.2 [16] The essential spectrum of $L_{0}$ on $H^{1}\left(S^{1}, \mathbf{R}^{N}\right)$ is just $\{-1,1\}$.

In view of the above facts, 0 is not in the essential spectrum of $L$ as it is a compact perturbation of the self-adjoint operator $L_{0}$. This implies that 0 is at most an eigenvalue of finite multiplicity of $L$.

Remark 2.1 $H^{1}\left(S^{1}, \mathbf{R}^{N}\right)$ has an orthogonal decomposition

$$
H^{1}\left(S^{1}, \mathbf{R}^{N}\right)=H^{+} \oplus H^{-} \oplus H^{0},
$$


where $H^{0}=\operatorname{Ker} L$ is finite dimensional, and $H^{+}, H^{-}$are $L$-invariant subspaces such that for some $\sigma>0$,

$$
\langle L u, u\rangle \geq \sigma\|u\|^{2}
$$

for all $u \in H^{+}$,

$$
\langle L u, u\rangle \leq-\sigma\|u\|^{2}
$$

for all $u \in H^{-}$.

Remark 2.2 By [16], we can choose the proper $r$ to make that the set $\left\{j \geq 0 \mid \frac{(-1)^{j} j^{2}-r \lambda^{2}}{j^{2}+1}=\right.$ $0\} \neq \emptyset$, which means $H^{0} \neq \emptyset$, where $j \in \mathbf{Z}$.

Lemma 2.3 [5] There exists $C>0$ such that

$$
\|u\|_{L^{p}} \leq C\|u\|, \quad\|u\|_{\infty} \leq C\|u\|
$$

for $p=1+\alpha, 2,\|u\|_{\infty}=\max _{t \in[0,2 \pi]}|u(t)|, \forall u \in H^{1}\left(S^{1}, \mathbf{R}^{N}\right)$.

Now consider the functional $\varphi$ defined on $H^{1}\left(S^{1}, \mathbf{R}^{N}\right)$ given by

$$
\varphi(u)=\frac{1}{2}\langle L u, u\rangle-\lambda^{2} \int_{0}^{2 \pi} F(\lambda t, u(t)) d t
$$

Since $F$ satisfies the assumption (A), a standard argument shows the following.

Lemma 2.4 [16] The functional $\varphi$ is continuously differentiable on $H^{1}\left(S^{1}, \mathbf{R}^{N}\right)$ and $\varphi^{\prime}$ is defined by

$$
\left\langle\varphi^{\prime}(u), v\right\rangle=\langle L u, v\rangle-\lambda^{2} \int_{0}^{2 \pi}(\nabla F(\lambda t, u(t)), v(t)) d t
$$

for all $v \in H^{1}\left(S^{1}, \mathbf{R}^{N}\right)$.

Lemma 2.5 [16] A critical point $u \in H^{1}\left(S^{1}, \mathbf{R}^{N}\right)$ of functional $\varphi$ is equivalent to a $2 \pi$ periodic solution of system (2.1).

In this paper, we will use the following local linking Theorem A to prove our theorems. The following concepts appeared in $[4,7,8]$.

Let $X$ be a real Banach space with direct decomposition $X=X^{1} \oplus X^{2}$. Consider two sequences of subspaces:

$$
X_{0}^{j} \subset X_{1}^{j} \subset \cdots \subset X^{j}
$$

such that $X^{j}=\overline{\bigcup_{n \in N} X_{n}^{j}}, j=1,2$. For every multi-index $\alpha=\left(\alpha_{1}, \alpha_{2}\right) \in \mathbf{N}^{2}$, we denote by $X_{\alpha}$ the space $X_{\alpha_{1}} \oplus X_{\alpha_{2}}$. We say $\alpha \leq \beta$ if $\alpha_{1} \leq \beta_{1}, \alpha_{2} \leq \beta_{2}$. 
Definition 2.1 [4] A sequence $\left\{\alpha_{n}\right\} \subset N^{2}$ is said to be admissible if, for every $\alpha \in \mathbf{N}^{2}$ there is $m \in N$ such that $n \geq m \Rightarrow \alpha_{n}>\alpha$.

Definition 2.2 [4] Let $f \in C^{1}(X, R)$. Then the function $f$ satisfies the $(P S)^{*}$ condition if every sequence $\left\{u_{\alpha_{n}}\right\}$ such that $\left\{\alpha_{n}\right\}$ is admissible and

$$
u_{\alpha_{n}} \in X_{\alpha_{n}}, \quad \sup _{n} f\left(u_{\alpha_{n}}\right)<\infty, \quad f_{\alpha_{n}}^{\prime}\left(u_{\alpha_{n}}\right) \rightarrow 0
$$

contains a subsequence which converges to a critical point of $f$, where $f_{\alpha}=\left.f\right|_{X_{\alpha}}$.

Definition 2.3 [4] Let $X$ be a Banach space with a direct sum decomposition $X=X^{1} \oplus X^{2}$. The function $f \in C^{1}(X, R)$ has a local linking at 0 , with respect to $\left(X^{1}, X^{2}\right)$, if, for some $r>0$,

$$
\begin{aligned}
& f(x) \geq 0, \quad \forall x \in X^{1},\|x\| \leq r, \\
& f(x) \leq 0, \quad \forall x \in X^{2},\|x\| \leq r .
\end{aligned}
$$

Theorem A [4] Suppose that $f \in C^{1}(X, \mathbf{R})$ satisfies the following assumptions:

(f1) $f$ has a local linking at 0 and $X^{1} \neq\{0\}$;

(f2) $f$ satisfies $(P S)^{*}$ condition;

(f3) $f$ maps bounded sets into bounded sets;

(f4) for every $m \in N, f(x) \rightarrow-\infty$ as $\|x\| \rightarrow \infty$ on $X_{m}^{1} \oplus X^{2}$.

Then $f$ has at least one nonzero critical point.

\section{Proofs of theorems}

In this section, $c_{i}$ stand for different positive constants for $i \in \mathbf{Z}^{+}, \mathbf{Z}^{+}$is the set of all positive integers.

Let

$$
X=X^{1} \oplus X^{2},
$$

where $X^{1}=H^{+}, X^{2}=H^{-} \oplus H^{0}$.

Lemma 3.1 Under assumption (H2), (H4)-(H5), the functional $\varphi$ satisfies the (PS)* condition.

Proof Let $\left\{u_{\alpha_{n}}\right\}$ be a sequence such that $\left\{\alpha_{n}\right\}$ is admissible and

$$
u_{\alpha_{n}} \in X_{\alpha_{n}}, \quad \sup _{n} \varphi\left(u_{\alpha_{n}}\right)<\infty, \quad \varphi_{\alpha_{n}}^{\prime}\left(u_{\alpha_{n}}\right) \rightarrow 0
$$

For the sake of notational simplicity, set $u_{n}=u_{\alpha_{n}}$.

Claim $1 .\left\{u_{n}\right\}$ is bounded in $X$.

If not, passing to a subsequence if necessary, we assume that $\left\|u_{n}\right\| \rightarrow \infty$ as $n \rightarrow \infty$. Set $v_{n}=\frac{u_{n}}{\left\|u_{n}\right\|}$, then $\left\{v_{n}\right\}$ is bounded in $X$. Hence, there exists a subsequence, still denoted by $\left\{v_{n}\right\}$. Write $v_{n}=v_{n}^{+}+v_{n}^{-}+v_{n}^{0}$ and $v=v^{+}+v^{-}+v^{0}$, then

$$
v_{n} \rightarrow v, \quad v_{n}^{0} \rightarrow v^{0} \quad \text { in } X, \quad v_{n} \rightarrow v \quad \text { in } C\left(S^{1}, \mathbf{R}^{N}\right) .
$$


In view of (H4), we have

$$
|F(t, x)| \leq c_{1}\left(|x|^{1+\alpha}+|x|\right), \quad \forall x \in \mathbf{R}^{N} \text { and } t \in[0,2 \tau] .
$$

For $|x| \leq R$ and $t \in[0,2 \tau]$, one has

$$
|F(t, x)| \leq c_{1}\left(|x|^{1+\alpha}+|x|\right) \leq c_{1}\left(R^{1+\alpha}+|R|\right):=A,
$$

together with (H5), one has

$$
\mu F(t, x) \leq(\nabla F(t, x), x)+b(t)|x|^{\beta}+\mu A, \quad \forall x \in \mathbf{R}^{N} \text { and } t \in[0,2 \tau] .
$$

Hence, by (2.6) and (2.7), we have

$$
\begin{aligned}
\left(\frac{\mu}{2}-1\right)\left\langle L u_{n}, u_{n}\right\rangle & =\mu \varphi\left(u_{n}\right)-\left\langle\varphi^{\prime}\left(u_{n}\right), u_{n}\right\rangle+\lambda^{2} \int_{0}^{2 \pi}\left[\mu F\left(\lambda t, u_{n}\right)-\left(\nabla F\left(\lambda t, u_{n}\right), u_{n}\right)\right] d t \\
& \leq c_{2}+c_{2}\left\|u_{n}\right\|+\lambda^{2} \int_{0}^{2 \pi}\left[b(t)\left|u_{n}\right|^{\beta}+\mu A\right] d t \\
& \leq c_{2}+c_{2}\left\|u_{n}\right\|+c_{3}\left\|u_{n}\right\|^{\beta}+c_{3} .
\end{aligned}
$$

This implies that

$$
\left\langle L u_{n}, u_{n}\right\rangle \leq c_{4}+c_{4}\left\|u_{n}\right\|+c_{4}\left\|u_{n}\right\|^{\beta}
$$

since $\mu>2$. Together with (3.4), we have

$$
\left\langle L v_{n}, v_{n}\right\rangle=\frac{\left\langle L u_{n}, u_{n}\right\rangle}{\left\|u_{n}\right\|^{2}} \rightarrow 0, \quad \text { as } n \rightarrow \infty,
$$

since $0<\beta<2$. Hence,

$$
\langle L v, v\rangle=0 .
$$

That means that

$$
\left\langle L v^{+}, v^{+}\right\rangle+\left\langle L v^{-}, v^{-}\right\rangle=0 .
$$

By using (2.3) and (2.4), we discuss two cases:

If $\left\|v^{+}\right\|=0$, we have $\left\|v^{-}\right\|=0$, then we obtain $\|v\|=\left\|v^{0}\right\|=\left\|v_{n}\right\|=1$.

If $\left\|v^{+}\right\| \neq 0$, we have $\left\|v^{-}\right\| \neq 0$, then we obtain $\|v\| \neq 0$.

So we get $v(t) \not \equiv 0$ for $t \in[0,2 \pi]$. Set

$$
E_{0}=\{t \in[0,2 \pi]: v(t) \neq 0\},
$$

then meas $\left(E_{0}\right)>0$. 
From (H2), we get

$$
\begin{aligned}
& \lim _{\left|u_{n}\right| \rightarrow \infty} \inf \frac{\int_{0}^{2 \pi} F\left(\lambda t, u_{n}\right) d t}{\left\|u_{n}\right\|^{2}} \\
& \quad \geq \frac{\int_{0}^{2 \pi}\left[\lim _{\left|u_{n}\right| \rightarrow \infty} \inf F\left(\lambda t, u_{n}\right)\right] d t}{\left\|u_{n}\right\|^{2}} \\
& \quad \geq \int_{E_{0}}\left[\lim _{\left|u_{n}\right| \rightarrow \infty} \inf \frac{F\left(\lambda t, u_{n}\right)}{\left|u_{n}\right|^{2}}\left|v_{n}\right|^{2}\right] d t \geq \int_{E_{0}}\left[\lim _{\left|u_{n}\right| \rightarrow \infty} \inf \frac{F\left(\lambda t, u_{n}\right)}{\left|u_{n}\right|^{2}}|v|^{2}\right] d t>0 .
\end{aligned}
$$

By the boundedness of $\varphi\left(u_{n}\right)$ and (3.4), we have

$$
\frac{\varphi\left(u_{n}\right)}{\left\|u_{n}\right\|^{2}}=\frac{\left\langle L u_{n}, u_{n}\right\rangle}{2\left\|u_{n}\right\|^{2}}-\frac{\lambda^{2} \int_{0}^{2 \pi} F\left(\lambda t, u_{n}\right) d t}{\left\|u_{n}\right\|^{2}} \leq \frac{c_{4}+c_{4}\left\|u_{n}\right\|+c_{4}\left\|u_{n}\right\|^{\beta}}{2\left\|u_{n}\right\|^{2}}-\frac{\lambda^{2} \int_{0}^{2 \pi} F\left(\lambda t, u_{n}\right) d t}{\left\|u_{n}\right\|^{2}}
$$

which together with $0<\beta<2$ implies that

$$
\lim _{\left|u_{n}\right| \rightarrow \infty} \inf \frac{\int_{0}^{2 \pi} F\left(\lambda t, u_{n}\right) d t}{\left\|u_{n}\right\|^{2}}=0
$$

This contradicts (3.5). Therefore, $\left\{u_{n}\right\}$ is bounded in $X$.

Claim 2. $\left\{u_{n}\right\}$ possesses a strong convergent subsequence in $X$.

Write $u_{n}=u_{n}^{+}+u_{n}^{-}+u_{n}^{0}$ and $u=u^{+}+u^{-}+u^{0}$, then

$$
u_{n}^{ \pm} \rightarrow u^{ \pm}, \quad u_{n}^{0} \rightarrow u^{0} \quad \text { in } X, \quad u_{n}^{ \pm} \rightarrow u^{ \pm} \quad \text { in } C\left(S^{1}, \mathbf{R}^{N}\right) .
$$

In view of (3.2) and $u_{n}^{-} \rightarrow u^{-}$in $C\left(S^{1}, \mathbf{R}^{N}\right)$, it is easy to verify

$$
\int_{0}^{2 \pi}\left(\nabla F\left(\lambda t, u_{n}\right)-\nabla F(\lambda t, u), u_{n}^{-}-u^{-}\right) d t \rightarrow 0, \quad \text { as } n \rightarrow \infty
$$

Note that

$$
\left\langle\varphi^{\prime}\left(u_{n}\right)-\varphi^{\prime}(u), u_{n}^{-}-u^{-}\right\rangle \rightarrow 0, \quad \text { as } n \rightarrow \infty
$$

Thus,

$$
\begin{aligned}
& -\left\langle\varphi^{\prime}\left(u_{n}\right)-\varphi^{\prime}(u), u_{n}^{-}-u^{-}\right\rangle \\
& =-\left\langle L\left(u_{n}^{-}-w^{-}\right), u_{n}^{-}-u^{-}\right\rangle+\lambda^{2} \int_{0}^{2 \pi}\left(\nabla F\left(\lambda t, u_{n}\right)-\nabla F(\lambda t, u), u_{n}^{-}-u^{-}\right) d t \\
& \geq \sigma\left\|u_{n}^{-}-u^{-}\right\|^{2}+\lambda^{2} \int_{0}^{2 \pi}\left(\nabla F\left(\lambda t, u_{n}\right)-\nabla F(\lambda t, u), u_{n}^{-}-u^{-}\right) d t .
\end{aligned}
$$

This yields $u_{n}^{-} \rightarrow u^{-}$in $X$. Similarly, $u_{n}^{+} \rightarrow u^{+}$in $X$. Hence $u_{n} \rightarrow u$ in $X$. Hence, $\left\{u_{n}\right\}$ possesses a strong convergent subsequence in $X$. The proof of Lemma 3.1 is complete.

Lemma 3.2 Under assumption (H2), (H4), and (H5'), the functional $\varphi$ satisfies the $(P S)^{*}$ condition. 
Proof Let $\left\{u_{\alpha_{n}}\right\}$ be a sequence such that $\left\{\alpha_{n}\right\}$ is admissible and

$$
u_{\alpha_{n}} \in X_{\alpha_{n}}, \quad \sup _{n} \varphi\left(u_{\alpha_{n}}\right)<\infty, \quad \varphi_{\alpha_{n}}^{\prime}\left(u_{\alpha_{n}}\right) \rightarrow 0 .
$$

For the sake of notational simplicity, set $u_{n}=u_{\alpha_{n}}$.

Claim $1 .\left\{u_{n}\right\}$ is bounded in $X$.

If not, passing to a subsequence if necessary, we assume that $\left\|u_{n}\right\| \rightarrow \infty$ as $n \rightarrow \infty$. In view of $\left(\mathrm{H} 5^{\prime}\right)$, there exists $c_{5}>0$ such that

$$
(\nabla F(t, x), x)-2 F(t, x) \geq b|x|^{\beta}-c_{5}, \quad \forall(t, x) \in[0,2 \tau] \times \mathbf{R}^{N} .
$$

Hence, we have

$$
\begin{aligned}
2 \varphi\left(u_{n}\right)-\left\langle\varphi^{\prime}\left(u_{n}\right), u_{n}\right\rangle & =\lambda^{2} \int_{0}^{2 \pi}\left[\left(\nabla F\left(\lambda t, u_{n}\right), u_{n}\right)-2 F\left(\lambda t, u_{n}\right)\right] d t \\
& \geq \lambda^{2} \int_{0}^{2 \pi}\left[b\left|u_{n}\right|^{\beta}-c_{5}\right] d t \\
& =b \lambda^{2} \int_{0}^{2 \pi}\left|u_{n}\right|^{\beta} d t-2 \pi c_{5} \lambda^{2} .
\end{aligned}
$$

This implies that

$$
\frac{\int_{0}^{T}\left|u_{n}\right|^{\beta} d t}{\left\|u_{n}\right\|} \rightarrow 0, \quad \text { as } n \rightarrow \infty .
$$

Let $u_{n}=u_{n}^{+}+u_{n}^{-}+u_{n}^{0} \in H^{+} \oplus H^{-} \oplus H^{0}$. By (H4), we have

$$
\begin{aligned}
\left\langle\varphi^{\prime}\left(u_{n}\right), u_{n}^{+}\right\rangle & =\left\langle L u_{n}^{+}, u_{n}^{+}\right\rangle-\lambda^{2} \int_{0}^{2 \pi}\left(\nabla F\left(\lambda t, u_{n}\right), u_{n}^{+}\right) d t \\
& \geq \sigma\left\|u_{n}^{+}\right\|^{2}-a \lambda^{2} \int_{0}^{2 \pi}\left(\left|u_{n}\right|^{\alpha}\left|u_{n}^{+}\right|+\left|u_{n}^{+}\right|\right) d t \\
& \geq \sigma\left\|u_{n}^{+}\right\|^{2}-a \lambda^{2}(2 \pi)^{\frac{\beta-\alpha}{\beta}}\left(\int_{0}^{2 \pi}\left|u_{n}\right|^{\alpha \times \frac{\beta}{\alpha}} d t\right)^{\frac{\alpha}{\beta}}\left\|u_{n}^{+}\right\|_{\infty}-2 \pi a \lambda^{2}\left\|u_{n}^{+}\right\|_{\infty} \\
& \geq \sigma\left\|u_{n}^{+}\right\|^{2}-a \lambda^{2}(2 \pi)^{\frac{\beta-\alpha}{\beta}} C\left\|u_{n}\right\|_{L^{\beta}}^{\alpha}\left\|u_{n}^{+}\right\|-2 \pi a \lambda^{2} C\left\|u_{n}^{+}\right\|
\end{aligned}
$$

for all $n$, where we use the Hölder inequality and (2.5). We have

$$
\frac{\left\|u_{n}^{+}\right\|}{\left\|u_{n}\right\|} \rightarrow 0, \quad \text { as } n \rightarrow \infty
$$

Similarly for $u_{n}^{-}$, we also get

$$
\frac{\left\|u_{n}^{-}\right\|}{\left\|u_{n}\right\|} \rightarrow 0, \quad \text { as } n \rightarrow \infty \text {. }
$$

Again, by $\left(\mathrm{H} 5^{\prime}\right)$, we have

$$
(\nabla F(t, x), x)-2 F(t, x) \geq b|x|-c_{6}, \quad \forall|x| \geq R>1, t \in[0,2 \tau],
$$


since $\beta>\alpha \geq 1$. Since $\operatorname{dim} H^{0}<\infty$,

$$
\begin{aligned}
2 \varphi\left(u_{n}\right)-\left\langle\varphi^{\prime}\left(u_{n}\right), u_{n}\right\rangle & =\lambda^{2} \int_{0}^{2 \pi}\left[\left(\nabla F\left(\lambda t, u_{n}\right), u_{n}\right)-2 F\left(\lambda t, u_{n}\right)\right] d t \\
& \geq \lambda^{2} \int_{0}^{2 \pi}\left[b\left|u_{n}\right|-c_{6}\right] d t \\
& \geq \lambda^{2} \int_{0}^{2 \pi}\left(b\left|u_{n}^{0}\right|-b\left|u_{n}^{+}\right|-b\left|u_{n}^{-}\right|-c_{6}\right) d t \\
& \geq c_{7}\left\|u_{n}^{0}\right\|-c_{7}\left\|u_{n}^{+}\right\|-c_{7}\left\|u_{n}^{-}\right\|-c_{7} .
\end{aligned}
$$

So we get

$$
\frac{\left\|u_{n}^{0}\right\|}{\left\|u_{n}\right\|} \rightarrow 0, \quad \text { as } n \rightarrow \infty
$$

Hence by (3.8)-(3.10), one has

$$
1=\frac{\left\|u_{n}\right\|}{\left\|u_{n}\right\|} \leq \frac{\left\|u_{n}^{0}\right\|+\left\|u_{n}^{+}\right\|+\left\|u_{n}^{-}\right\|}{\left\|u_{n}\right\|} \rightarrow 0, \quad \text { as } n \rightarrow \infty
$$

a contradiction. Therefore, $\left\{u_{n}\right\}$ is bounded in $X$.

Using similar arguments to the proof of Claim 2 in Lemma 3.1, $\left\{u_{n}\right\}$ possesses a strong convergent subsequence in $X$. The proof of Lemma 3.2 is complete.

Lemma 3.3 Under assumption (H1)-(H4), the function $\varphi$ satisfies the conditions (f1), (f3), and (f4) of Theorem A.

Proof We only consider the case (i) in (H1). The other case is similar.

(1) We claim that $\varphi$ has a local linking at 0 with respect to $\left(X^{1}, X^{2}\right)$.

By (H3), for any $\varepsilon>0$, there exists $\delta>0$ such that

$$
|F(t, x)| \leq \varepsilon|x|^{2}, \quad \forall t \in[0,2 \tau],|x| \leq \delta
$$

By (3.2) and (3.11), there exists $c_{8}>0$ such that

$$
|F(t, x)| \leq \varepsilon|x|^{2}+c_{8}|x|^{1+\alpha}, \quad \forall t \in[0,2 \tau], x \in \mathbf{R}^{N} .
$$

Hence, for $u \in X^{1}$, we have

$$
\varphi(u) \geq \frac{1}{2}\langle L u, u\rangle-\lambda^{2} \int_{0}^{2 \pi}\left(\varepsilon|u|^{2}+c_{8}|u|^{1+\alpha}\right) d t \geq \frac{\sigma}{2}\|u\|^{2}-\varepsilon \lambda^{2} C\|u\|^{2}-c_{8} \lambda^{2} C\|u\|^{1+\alpha} .
$$

Taking $\varepsilon=\sigma /\left(4 \lambda^{2} C\right)$ and noting that $\alpha>1$ in (H4), we can find a constant $\rho_{1}>0$ such that

$$
\varphi(u) \geq 0, \quad \forall u \in X^{1} \text { with }\|u\| \leq \rho_{1} .
$$


On the other hand, let $u=u^{0}+u^{-} \in X^{2}$ satisfying $\|u\| \leq \rho_{0}=R_{1} /(2 C)$ and let

$$
\Omega_{1}=\left\{t \in[0,2 \pi]|| u^{-} \mid \leq R_{1} / 2\right\}, \quad \Omega_{2}=\left\{t \in[0,2 \pi]|| u^{-} \mid>R_{1} / 2\right\} .
$$

Then we have

$$
\left|u^{0}\right| \leq\left\|u^{0}\right\|_{\infty} \leq C\left\|u^{0}\right\| \leq R_{1} / 2
$$

for all $t \in[0,2 \pi]$. Consequently,

$$
|u| \leq\left|u^{0}\right|+\left|u^{-}\right| \leq R_{1}, \quad \text { for all } t \in \Omega_{1} .
$$

Hence, from (H1)(i), we obtain

$$
\int_{\Omega_{1}} F(t, u) d t \geq 0
$$

On the one hand, one obtains

$$
|u| \leq\left|u^{0}\right|+\left|u^{-}\right| \leq R_{1} / 2+\left|u^{-}\right| \leq 2\left|u^{-}\right|, \quad \text { for all } t \in \Omega_{2} .
$$

It follows from (3.12) that

$$
|F(\lambda t, u)| \leq \varepsilon|u|^{2}+c_{8}|u|^{1+\alpha} \leq 4 \varepsilon\left|u^{-}\right|^{2}+2^{1+\alpha} c_{8}\left|u^{-}\right|^{1+\alpha},
$$

for all $t \in \Omega_{2}$, which implies that

$$
\begin{aligned}
\left|\int_{\Omega_{2}} F(\lambda t, u) d t\right| & \leq 4 \varepsilon \int_{\Omega_{2}}\left|u^{-}\right|^{2} d x+2^{1+\alpha} c_{8} \int_{\Omega_{2}}\left|u^{-}\right|^{1+\alpha} d t \\
& \leq 4 \varepsilon \int_{0}^{2 \pi}\left|u^{-}\right|^{2} d x+2^{1+\alpha} c_{8} \int_{0}^{2 \pi}\left|u^{-}\right|^{1+\alpha} d t \\
& \leq 4 \varepsilon C^{2}\left\|u^{-}\right\|^{2}+(2 C)^{1+\alpha} c_{8}\left\|u^{-}\right\|^{1+\alpha} .
\end{aligned}
$$

Setting $\varepsilon=\sigma /\left(16 C^{2}\right)$, we have

$$
\varphi(u) \leq-\frac{\sigma}{2}\left\langle u^{-}, u^{-}\right\rangle-\lambda^{2} \int_{0}^{2 \pi} F(\lambda t, u) d t \leq-\frac{\sigma}{4}\left\|u^{-}\right\|^{2}+(2 C)^{1+\alpha} c_{8}\left\|u^{-}\right\|^{1+\alpha} .
$$

Consequently,

$$
\varphi(u) \leq 0, \quad \forall u \in X^{2} \text { with }\|u\| \leq \rho_{2},
$$

where $\rho_{2}<\rho_{0}$ is small enough.

(2) We claim that for every $m \in \mathbf{N}, \varphi(u) \rightarrow-\infty$ as $\|u\| \rightarrow \infty$ on $X_{m}^{1} \oplus X^{2}$.

Since $X_{m}^{1}$ and $H^{0}$ are finite dimensional, we can choose $\sigma_{m}>0, C_{m}>0$ such that

$$
\left\langle L u^{+}, u^{+}\right\rangle \leq \sigma_{m}\left\|u^{+}\right\|, \quad \text { for all } u^{+} \in X_{m}^{1},
$$




$$
\|u\| \leq C_{m}\|u\|_{L^{2}}, \quad \text { for all } u \in X_{m}^{1} \oplus H^{0} .
$$

By $(\mathrm{H} 2)$, there exists $c_{9}>0$ such that

$$
F(t, x) \geq \frac{\sigma_{m}}{C_{m}}|x|^{2}-c_{9}, \quad \forall t \in[0,2 \tau], \forall x \in \mathbf{R}^{N} .
$$

So for $u \in X_{m}^{1} \oplus X^{2}$,

$$
\begin{aligned}
\varphi(u) & =\frac{1}{2}\left\langle L u^{+}, u^{+}\right\rangle+\frac{1}{2}\left\langle L u^{-}, u^{-}\right\rangle-\lambda^{2} \int_{0}^{2 \pi} F(\lambda t, u(t)) d t \\
& \leq \frac{\sigma_{m}}{2}\left\|u^{+}\right\|^{2}-\frac{\sigma}{2}\left\|u^{-}\right\|^{2}-\frac{\sigma_{m}}{C_{m}} \int_{0}^{2 \pi}|u(t)|^{2} d t+c_{10} \\
& =\frac{\sigma_{m}}{2}\left\|u^{+}\right\|^{2}-\frac{\sigma}{2}\left\|u^{-}\right\|^{2}-\frac{\sigma_{m}}{C_{m}}\left(\left\|u^{+}\right\|_{L^{2}}+\left\|u^{-}\right\|_{L^{2}}+\left\|u^{0}\right\|_{L^{2}}\right)+c_{10} \\
& \leq-\frac{\sigma_{m}}{2}\left\|u^{+}\right\|^{2}-\frac{\sigma}{2}\left\|u^{-}\right\|^{2}-\sigma_{m}\left\|u^{0}\right\|+c_{10} \rightarrow-\infty,
\end{aligned}
$$

for $\|u\| \rightarrow \infty$.

(3) By (3.2), we see that $\varphi$ maps bounded sets into bounded sets.

The proof of Lemma 3.3 is complete.

Proof of Theorem 1.1 By Lemmas 3.1 and 3.3, all conditions of Theorem A are satisfied. Thus, problem (2.1) has at least one nonzero critical point.

Proof of Theorem 1.2 By Lemmas 3.2 and 3.3, all conditions of Theorem A are satisfied. Thus, problem (2.1) has at least one nonzero critical point.

\section{Competing interests}

The authors declare that they have no competing interests.

\section{Authors' contributions}

The authors read and approved the final manuscript.

\section{Acknowledgements}

The authors would like to express their sincere thanks to the referees for their helpful comments. Supported by the Natural Science Foundation of Shanxi Province (No. 2012011004-1) of China and by the National Natural Science Foundation of China (No. 61473180).

Received: 28 August 2015 Accepted: 25 January 2016 Published online: 05 February 2016

\section{References}

1. Ding, Y: Variational Methods for Strongly Indefinite Problems. World Scientific, Singapore (2007)

2. Guo, Y: Nontrivial periodic solutions for asymptotically linear Hamiltonian systems with resonance. J. Differ. Equ. 175 71-87 (2001)

3. Su, JB: Nontrivial periodic solutions for the asymptotically linear Hamiltonian systems with resonance at infinity. J. Differ. Equ. 145, 252-273 (1998)

4. Li, SJ, Willem, M: Applications of local linking to critical point theory. J. Math. Anal. Appl. 189, 6-32 (1995)

5. Mawhin, J, Willem, M: Critical Point Theory and Hamiltonian Systems. Springer, New York (1989)

6. Rabinowitz, PH: Minimax Methods in Critical Point Theory with Applications to Differential Equations. CBMS Reg. Conf. Ser. Math., vol. 65. Am. Math. Soc., Providence (1986)

7. Zhang, Q, Tang, XH: New existence of periodic solutions for second order non-autonomous Hamiltonian systems. J. Math. Anal. Appl. 369, 357-367 (2010)

8. He, X, Wu, X: Periodic solutions for a class of nonautonomous second order Hamiltonian systems. J. Math. Anal. Appl. $341,1354-1364(2008)$

9. Fei, G: Multiple periodic solutions of differential delay equations via Hamiltonian systems (I). Nonlinear Anal. 65, 25-39 (2006)

10. Fei, G: Multiple periodic solutions of differential delay equations via Hamiltonian systems (II). Nonlinear Anal. 65 40-58 (2006) 
11. Guo, Z, Yu, J: Multiplicity results for periodic solutions to delay differential equations via critical point theory. J. Differ. Equ. 218, 15-35 (2005)

12. $\mathrm{Li}, \mathrm{J}, \mathrm{He}, \mathrm{X}$ : Multiple periodic solutions of differential delay equations created by asymptotically linear Hamiltonian systems. Nonlinear Anal. 31(1/2), 45-54 (1998)

13. $\mathrm{Wu}, \mathrm{K}, \mathrm{Wu}, \mathrm{X}$ : Existence of periodic solutions for a class of first order delay differential equations dealing with vectors. Nonlinear Anal. 72, 4518-4529 (2010)

14. Wu, K, Wu, X: Multiplicity results of periodic solutions for systems of first order delay differential equation. Appl. Math. Comput. 218, 1765-1773 (2011)

15. Wu, K: Multiplicity results of periodic solutions for a class of first order delay differential equations. J. Math. Anal. Appl. $390,427-438$ (2012)

16. Wu, K, Wu, X, Zhou, F: Multiplicity results of periodic solutions for a class of second order delay differential equations. Nonlinear Anal. 75, 5836-5844 (2012)

17. Zhang, X, Meng, Q: Nontrivial periodic solutions for delay differential systems via Morse theory. Nonlinear Anal. 74, 1960-1968 (2011)

18. $\mathrm{Yu}, \mathrm{J}, \mathrm{XiaO}, \mathrm{H}$ : Multiplicity periodic solutions with minimal period 4 of delay differential equations $x^{\prime}(t)=-f(t, x(t-1))$. J. Differ. Equ. 254, 2158-2172 (2013)

\section{Submit your manuscript to a SpringerOpen ${ }^{\circ}$ journal and benefit from:}

- Convenient online submission

- Rigorous peer review

- Immediate publication on acceptance

- Open access: articles freely available online

- High visibility within the field

- Retaining the copyright to your article 